\title{
MRI-based or CT-based patient-specific instrumentation in Total knee Arthroplasty: How do the two systems compare?
}

\author{
Dong-Geun Kang, Kang-II Kim *i] and Jung-Kwon Bae
}

\begin{abstract}
Background: Patient-specific instrumentation (PSI) has been introduced into total knee arthroplasty (TKA) to improve accuracy in restoration of alignment. PSI in TKA refers to custom-made cutting jigs manufactured according to anatomic configuration of the patient's bone based on preoperative magnetic resonance imaging (MRI) or computed tomography (CT) scans. The purpose of this study was to compare the MRI- or CT-based PSI to see if they could reproduce accurate bone resection and postoperative outcomes.
\end{abstract}

Methods: Seventy-one patients who received elective TKA using a PSI system for primary osteoarthritis with varus deformity were prospectively enrolled for this study. We randomly allocated those patients to MRI-based PSI group (36 patients) and CT-based PSI group (35 patients). The actual resection thickness and planned resection thickness by preoperative PSI electronic program were compared between the two groups. Radiographic findings of the postoperative limb alignment, three-dimensional position of the implants, and related complications were also evaluated. Clinical evaluation was also performed before and 2 years after the surgery.

Results: There were no significant differences in the resection thickness in femur and tibia between actual resection and planned resection in both groups. Furthermore, there were no significant differences between two groups in terms of coronal, sagittal and rotational alignment of the components. All clinical assessments revealed no differences between two groups 2 years after the operation. No specific complication related to PSI was observed.

Conclusions: Although MRI allows for visualization of cartilage, MRI-based PSI system did not show better accuracy in predicting the thickness of bone resection than CT-based PSI. Moreover, there were no differences in radiographic and clinical outcomes between the two groups.

Keywords: Total knee arthroplasty, Patient specific instrumentation, Magnetic resonance image, Computed tomography

\section{Background}

Patient-specific instrumentation (PSI) has been introduced into total knee arthroplasty (TKA) as a new technology for improving accuracy in restoration of alignment and biomechanics of the lower limb [1-4]. PSI in TKA refers to custom-made cutting jigs manufactured according to the patient's anatomic configuration of distal femur and proximal tibia based on preoperative magnetic resonance imaging (MRI) or computed tomography (CT) scans [5-8]. MRI- and CT-based PSI systems are available from various

\footnotetext{
* Correspondence: khuknee@gmail.com

Department of Orthopaedic Surgery, Kyung Hee University Hospital at Gangdong, 892 Dongnam-ro, Gangdong-gu, Seoul 134-727, South Korea
}

manufacturers for preoperative planning. MRI gives concrete form to articular cartilage without the risk of radiation exposure, but it is relatively expensive and requires longer scan time than $\mathrm{CT}$. In contrast, CT enables accurate identification of the contour of the femur and tibia for shorter scan time [9], but it cannot provide information on the articular cartilage and carries the risk of radiation exposure. As a result, there might be some discrepancies between the thickness of bone resection proposed by MRI- or CT-based PSI system and the actual thickness of bone cutting. MRI has the theoretical advantage of full visualization of articular cartilage and is supposed to be more accurate in predicting bone resection thickness in TKA than the CT-based

(c) The Author(s). 2020 Open Access This article is distributed under the terms of the Creative Commons Attribution 4.0 International License (http://creativecommons.org/licenses/by/4.0/), which permits unrestricted use, distribution, and 
PSI system. Previous studies compared PSI with the conventional instrumentation and computer-assisted navigation system in terms of its efficacy in improving the accuracy of TKA [10-12]. Although PSI has been the focus of many recent researches $[6,12-16]$, there have been a few clinical studies comparing MRI- and CT-based PSI systems in preoperative planning [17-19]. However, to our knowledge, this would be the first study to examine the accuracy of bone resection by preoperative planning for two imaging modalities in PSI system. The purpose of this study was to know whether the MRI- or CT-based PSI could reproduce accurate bone resection and better postoperative outcomes. Therefore, we tried to know whether the MRIbased PSI that reflects the cartilage layer would provide more precision in TKA than the CT-based PSI. We hypothesized the MRI-based PSI would be more accurate for resection thickness of lateral aspect of proximal tibia where the cartilage is relatively sound in varus knee and the two systems would have no difference in the resection of the medial femoral condyle where the cartilage would be generally worn in varus knee. In addition, we compared the radiographic findings and clinical outcomes between the groups.

\section{Methods}

\section{Study design}

This was a prospective study of PSI-guided TKAs performed by a single surgeon (KIK). The institutional review board granted approval for this study. Written informed consent was obtained from all patients before the surgery. Of the patients who had been scheduled for TKA for the treatment of primary osteoarthritis only with varus deformity, those who had been waiting 6 weeks for TKA using an MRI-based or CT-based PSI system and had consented to the relatively new technique were enrolled. Patients with primary osteoarthritis with valgus deformity, rheumatoid arthritis, hemophilic arthritis, posttraumatic arthritis, other inflammatory arthritis, or a history of previous high tibial osteotomy were excluded from the study. Therefore, 71 TKA candidates were assigned, using a computer-derived randomization table with block sizes of four, to receive an MRI scan (MRI group; $N=36$ ) or a CT scan (CT group; $N=35$ ) (Fig. 1). In all patients, the Signature Personalized Patient Care System (Biomet Inc., Warsaw, Indiana) manufactured using Materialise ${ }^{\curvearrowleft}$ software (Leuven, Belgium) was used. There were no significant differences between the groups with regards to age, gender, body mass index, degree of preoperative deformity (Table 1 ). The followup assessment was conducted 2 years after the surgery in all patients without exception.

\section{Preoperative planning and PSI manufacturing}

In the MRI group, preoperative MRI data acquisition was performed using an MRI scanner (Achieva 3.0 T, Philips, Netherlands) according to the PSI system manufacturing protocol: 1-mm high-resolution slices of the knee and selective 5-mm spot images of the hip and ankle were acquired to measure the MA and to correct rotation of the lower extremity. The MRI images in DICOM format (Digital Imaging and Communications in Medicine) were uploaded onto the software to construct three-dimensional (3D) models. Then,

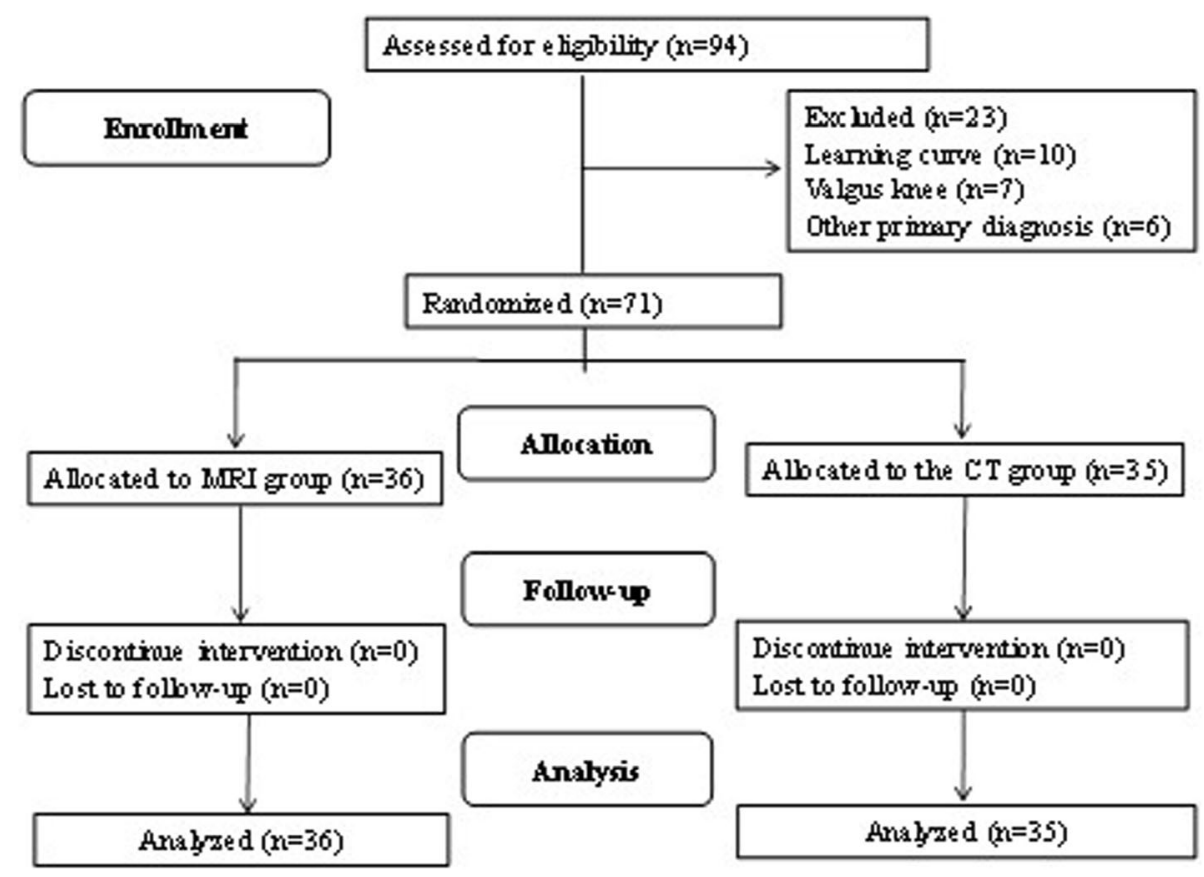

Fig. 1 CONSORT flow diagram of randomization and follow-up of patients 
Table 1 Demographic data

\begin{tabular}{llll}
\hline Parameter & MRI group $(N=36)$ & $C T$ group $(N=35)$ & $P$ Value \\
\hline Age $(\text { year })^{\mathrm{a}}$ & $69.1 \pm 5.9$ & $68.5 \pm 7.0$ & 0.685 \\
Sex $($ male: female) & $5: 31$ & $1: 34$ & 0.199 \\
BMI $\left(\mathrm{kg} / \mathrm{m}^{2}\right)^{\mathrm{a}}$ & $27.1 \pm 4.0$ & $27.1 \pm 3.3$ & 0.990 \\
Preoperative MA $\left(^{\circ}\right)^{\mathrm{a}}$ & $-9.9 \pm 4.8$ & $-10.4 \pm 4.8$ & 0.673 \\
Preoperative FTA $\left({ }^{\circ}\right)^{\mathrm{a}}$ & $-3.9 \pm 4.5$ & $-3.9 \pm 5.2$ & 0.963 \\
\hline
\end{tabular}

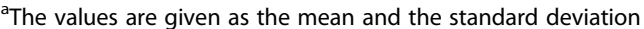
$B M I$ Body mass index, MA Mechanical axis, FTA Femorotibial angle

the patient-specific cutting jigs were generated based on the surgeon's predetermined default settings. The femoral component rotation was set to be parallel to the transepicondylar axis, perpendicular to the MA on the coronal plane, and in $3^{\circ}$ flexion on the sagittal plane, and medial distal resection was set at $9 \mathrm{~mm}$. The default settings for tibial component alignment were perpendicular to the MA on the coronal plane $\left(0^{\circ}\right.$ varus/valgus) and at a $3^{\circ}$ posterior slope on the sagittal plane, and tibial resection was set at $10 \mathrm{~mm}$ below the highest point of the lateral tibia. The tibial component rotation could not be adjusted by the software.

In the CT group, preoperative CT data acquisition was performed using a CT scanner (Brilliance 64 Channel Multi Detector Computed Tomography, Philips, Israel) according to the same PSI system manufacturing protocol: $1.25-\mathrm{mm}$ slices of the lower extremity were obtained to measure the mechanical axis (MA) and to correct rotation of the lower extremity. The CT images in DICOM format were processed by the same software to reconstruct 3D models. Since most varus knees in primary osteoarthritis have a relatively sound lateral compartment and a worn medial compartment, the default setting values were identical to those used for the MRI-based PSI in the medial bone cut, lateral cutting thickness of proximal tibia was set at $8 \mathrm{~mm}$ below the highest point of the lateral tibia. We presumed it would be $10 \mathrm{~mm}$ of tibial cutting by compensating for the cartilage which could not be seen on CT scans $[20,21]$. The predetermined values for the implant size, knee alignment, and thickness of bone resection in both groups were reviewed for potential adjustment. Upon approval of the preoperative plan, the PSI manufacturer was requested to produce cutting jigs and bone models designed to represent the patient's anatomical configuration of the femur and tibia. The bone models and cutting jigs were made of polyamide and fabricated using rapid prototyping [4].

\section{Operative technique}

All the operations were performed under general anesthesia and tourniquet control by a senior surgeon using a minimally invasive mid-vastus approach. The patient-specific cutting jigs were used for bone resection in all knees: without osteophyte removal, the cutting jigs were placed on the femur and the tibia, ensuring stable fitting without toggling. By using a modified gap technique, the distal femur was resected, which was followed by proximal tibial bone cutting and femoral chamfer resection by employing PSI cutting jigs. Except for the use of PSI instead of the conventional instrument for bone resection, overall surgical procedure was identical to the routine conventional method. All bone cuts were made using conventional $1.27-\mathrm{mm}$ saw blades. All patients received the posteriorstabilized cemented total knee system (Vanguard $^{\mathrm{jm}}$, Biomet, Warsaw, Indiana). A suction drain insertion, pneumatic pump application to prevent deep vein thrombosis, multimodal pain managements, and postoperative rehabilitation protocol were identical in all patients.

\section{Assessment}

After bone resection using a patient-specific cutting jig, the actual thickness of resected bone was measured in $0.1 \mathrm{~mm}$ increments by the Vernier caliper (B. BraunAesculap, Tuttlingen, Germany) and compared with the planned resection thickness preoperatively using the PSI program. In the MRI group, the intraoperative cutting thickness was compared directly with the preoperatively planned thickness, whereas the presumed thickness of cartilage $(2 \mathrm{~mm})$ was added to actual thickness of resected bone from the lateral condyles in the $\mathrm{CT}$ group $[20,21]$. Finally, the thickness of saw blade $(1.27-\mathrm{mm})$ was added to every resected bone in all cases.

On the radiographic assessment, the MA of the lower extremity and the coronal and sagittal alignments of the femoral and tibial components were measured on the anterior and lateral weight-bearing radiographs of the knee and the standing long-leg radiograph before the surgery and 2 years after the surgery. The measurements were performed using a picture-acquiring communication system (PACS, Infinitt Healthcare, Seoul, Korea), and the data were recorded to one decimal place. The MA was defined as the angle formed by the femoral MA (a line between the center of the femoral head and the center of the knee) and the tibial MA (a line between the center of the ankle and the center of the knee): $0^{\circ}$ was regarded as neutral, a varus alignment was assigned a negative value, and a valgus alignment was assigned a positive value. The coronal femoral angle $(\alpha)$ and the coronal tibial angle $(\beta)$ were assessed on the anteroposterior weight-bearing radiograph, whereas the sagittal femoral angle $(\gamma)$ and the sagittal tibial angle $(\delta)$ were measured on the lateral radiograph [22]. The femoral component rotation was assessed on the postoperative 3D CT scan. The femoral component rotation was defined as the angle difference between the posterior condylar axis of the femoral component and the surgical epicondylar axis [23]. An outlier was defined as $\pm 3^{\circ}$ deviation from the ideal alignment. The tibial component rotation was not assessed postoperatively. We believed PSI would have no direct influence on the postoperative 
rotational alignment of the tibial component because it was not included in the preoperative planning and the optimal alignment was determined intraoperatively based on the assessment of knee flexion/extension after trial insertion. Measurements were performed by two blinded, independent observers, twice each, with a minimum interval of 2 weeks.

For clinical assessment, clinical scores before the surgery and 2 years after the surgery were compared between the two groups. Clinical assessment included the Knee Society knee score [24] and the 36-item Short Form Survey [25] in all patients.

\section{Statistical analysis}

Continuous variables including radiographic measurements, and differences in resected bone thickness were compared between groups with Student's $t$-test or Mann-Whitney U test (for data that were not normally distributed). Pearson's chi-square test, Fisher's exact test were used to compare categorical variables between the two groups, such as the incidence of outliers of MA and femoral component rotation. All statistical analyses were performed with SPSS for Windows, version 12.0 (SPSS, Chicago, Illinois). $P$ values $<0.05$ were considered statistically significant. Inter- and intraobserver reliability was assessed using the intraclass correlation coefficient (ICC). The ICCs were classified as follows: poor, less than 0.4; marginal, 0.4-0.75; and good, above 0.75.

\section{Results}

The absolute differences between the planned resection thickness and the actual resection thickness in the distal femur and the proximal tibia were not significantly different between the MRI group and the CT group (Table 2). The mean postoperative MA of the lower limb and the prevalence of outliers with reference to the MA did not differ between the two groups $(p>0.05)$ (Table 3). Moreover, there was no notable intergroup difference in the mean sagittal and coronal alignments of the femoral and tibial components 2 years after TKA. The mean rotational alignment of the femoral component was $1.2^{\circ}$ external rotation in the MRI group and $1.1^{\circ}$ external rotation in the CT group $(p>0.05)$. The incidences of outliers were also similar between the groups. The intraobserver ICC for each variable was 0.863 0.935 and the interobserver ICC was $0.807-0.836$, indicating high agreement between the observers and measurements. There was no difference in mean preoperative and postoperative Knee Society knee and functional scores, 36-item Short Form Survey between the two groups 2 years after surgery (Table 4). No patients required reoperation or rehospitalization following TKA, and there were no perioperative complications, including deep or superficial infection, symptomatic deep vein thrombosis or pulmonary embolisms, and periprosthetic fracture until the latest follow-up.

\section{Discussion}

This study demonstrated that there were no significant differences between the MRI- and CT- based PSI systems in following investigated parameters: 1) the differences between the planned resection thickness and the actual resection thickness in the distal femur and the proximal tibia; 2) the mean postoperative MA of the lower limb and the prevalence of outliers with reference to the MA; 3) the mean postoperative alignments and short-term clinical outcomes.

The differences between the planned resection thickness and the actual resection thickness in the distal femur and the proximal tibia were not significantly different in both studied groups in this study. In the current study, the primary osteoarthritic knee with varus deformity alone was an inclusion criterion. Therefore, we assumed that the medial femoral cartilage was mostly worn out, whereas the cartilage thickness in the lateral tibial condyle was relatively sound in all study participants. In the CT group, we added $2 \mathrm{~mm}$ of presumed cartilage thickness to the bone thickness of lateral tibial resection to compensate for the cartilage layer surrounding the subchondral bone that was not visualized on CT. In the MRI group, there was no need to make such adjustments because MRI had the ability to represent the

Table 2 Comparison of absolute differences between the planned and actual bone resections in both groups

\begin{tabular}{|c|c|c|c|c|c|c|c|}
\hline \multirow[t]{2}{*}{$(\mathrm{mm})$} & \multicolumn{3}{|c|}{ MRI group $(N=36)$} & \multicolumn{3}{|c|}{$C T$ group $(N=35)$} & \multirow{2}{*}{$\begin{array}{l}\text { MRI vs } C \\
P \text { Value }\end{array}$} \\
\hline & Planned & Actual & Difference $^{\dagger}$ & Planned & Actual & $\overline{\text { Difference }^{\dagger}}$ & \\
\hline DFM & $9.0 \pm 0.7$ & $8.5 \pm 1.2$ & $0.9 \pm 0.7$ & $8.7 \pm 0.9$ & $9.0 \pm 1.6$ & $1.2 \pm 0.7$ & 0.192 \\
\hline DFL & $9.1 \pm 1.1$ & $8.3 \pm 1.6$ & $1.4 \pm 0.9$ & $9.3 \pm 1.6$ & $8.4 \pm 1.9$ & $1.6 \pm 1.0$ & 0.292 \\
\hline PFM & $9.3 \pm 0.6$ & $9.9 \pm 1.3$ & $1.1 \pm 0.9$ & $8.8 \pm 0.5$ & $8.9 \pm 1.8$ & $1.4 \pm 0.9$ & 0.173 \\
\hline PFL & $7.8 \pm 1.4$ & $7.2 \pm 1.5$ & $1.3 \pm 0.9$ & $8.6 \pm 1.2$ & $7.3 \pm 1.5$ & $1.6 \pm 0.8$ & 0.062 \\
\hline TM & $2.5 \pm 1.8$ & $2.9 \pm 1.0$ & $1.1 \pm 0.9$ & $2.1 \pm 1.3$ & $2.7 \pm 0.8$ & $1.1 \pm 0.9$ & 0.902 \\
\hline $\mathrm{TL}$ & $10.0 \pm 0.4$ & $9.4 \pm 1.3$ & $1.3 \pm 0.8$ & $10.3 \pm 0.7$ & $9.5 \pm 1.4$ & $1.2 \pm 0.8$ & 0.730 \\
\hline
\end{tabular}

${ }^{*}$ The values are given as the mean and the standard deviation. ${ }^{\dagger}$ paired $t$-test

DFM Distal femoral medial side, DFL Distal femoral lateral side

PFM Posterior femoral medial side, PFL Posterior femoral lateral side

$T M$ Tibial medial side, $T L$ Tibial lateral side 
Table 3 Postoperative radiographic measurements

\begin{tabular}{|c|c|c|c|}
\hline Parameter & MRI group $(N=36)$ & CT group $(N=35)$ & $P$ Value \\
\hline$\overline{\text { Mechanical axis }(M A)^{a}}$ & $-2.1^{\circ} \pm 2.1^{\circ}$ & $-2.1^{\circ} \pm 2.5^{\circ}$ & 0.996 \\
\hline MA outlier $\left(>3^{\circ}\right)^{\mathrm{b}}$ & $7(19.4 \%)$ & $8(22.9 \%)$ & 0.778 \\
\hline Coronal femoral angle $(a)^{a}$ & $94.9^{\circ} \pm 1.1^{\circ}$ & $94.7^{\circ} \pm 2.0^{\circ}$ & 0.747 \\
\hline Coronal tibial angle $(\beta)^{\mathrm{a}}$ & $90.0^{\circ} \pm 2.0^{\circ}$ & $90.1^{\circ} \pm 1.5^{\circ}$ & 0.909 \\
\hline Sagittal femoral angle $(\gamma)^{a}$ & $5.0^{\circ} \pm 2.9^{\circ}$ & $4.3^{\circ} \pm 2.8^{\circ}$ & 0.282 \\
\hline Sagittal tibial angle $(\delta)^{a}$ & $86.2^{\circ} \pm 2.8^{\circ}$ & $85.6^{\circ} \pm 3.0^{\circ}$ & 0.420 \\
\hline Femoral external rotation (ER) ${ }^{a}$ to transepicondylar axis & $1.2^{\circ} \pm 1.1^{\circ}$ & $1.1^{\circ} \pm 1.4^{\circ}$ & 0.810 \\
\hline Femoral ER outlier $\left(>3^{\circ}\right)^{\mathrm{b}}$ & $1(2.8 \%)$ & $3(8.6 \%)$ & 0.357 \\
\hline
\end{tabular}

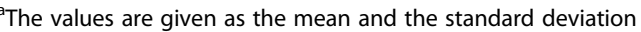

${ }^{b}$ Outlier values are given as the number of knees, with the percentage in parentheses

cartilage layer. The study showed no significant difference between the two groups in terms of the thickness of bone resection of the lateral aspect of the femur. Therefore, we recommend adding approximately $2 \mathrm{~mm}$ of cartilage thickness to the value proposed by the CTbased PSI system in determining the resection thickness of the lateral aspects of the tibia in patients with varus deformity [20, 21]. In both MRI and CT groups, about 1 $\mathrm{mm}$ of the mean absolute difference was noted between the planned resection thickness and the actual thickness of the resected bones on the worn medial side and the relatively intact lateral side, indicating that the accuracy of the PSI system was high. Furthermore, no statistically significant intergroup difference was observed, and the difference was within the margin of error. Hafez et al. [26] reported that the mean bone resection error was $0.32 \mathrm{~mm}$ (maximum, $1 \mathrm{~mm}$ ) in 16 cadavers and 29 plastic knee specimens after TKA using a CT-based PSI. This experimental result also supports our current study with in vivo settings. However, that study did not consider the thickness of invisible cartilage.

Table 4 Clinical outcomes between MRI- versus CT-based PSI after total knee arthroplasty

\begin{tabular}{|c|c|c|c|}
\hline Parameter & MRI group $(N=36)$ & $\mathrm{CT}$ group $(N=35)$ & $P$ Value \\
\hline \multicolumn{4}{|c|}{ Knee Society knee score } \\
\hline preoperative & $44.3 \pm 18.6$ & $42.8 \pm 16.1$ & 0.725 \\
\hline 2-year follow-up & $92.7 \pm 10.7$ & $92.2 \pm 11.8$ & 0.815 \\
\hline \multicolumn{4}{|c|}{ Knee Society function score } \\
\hline preoperative & $48.7 \pm 10.8$ & $46.9 \pm 10.4$ & 0.614 \\
\hline 2-year follow-up & $91.9 \pm 15.8$ & $89.7 \pm 12.9$ & 0.586 \\
\hline \multicolumn{4}{|c|}{ SF-36 (physical function) } \\
\hline preoperative & $30.9 \pm 9.7$ & $37.2 \pm 8.8$ & 0.436 \\
\hline 2-year follow-up & $47.5 \pm 8.5$ & $47.2 \pm 8.5$ & 0.539 \\
\hline \multicolumn{4}{|l|}{ SF-36 (mental health) } \\
\hline preoperative & $48.2 \pm 4.6$ & $50.7 \pm 5.3$ & 0.487 \\
\hline 2-year follow-up & $56.5 \pm 10.1$ & $60.8 \pm 11.9$ & 0.639 \\
\hline
\end{tabular}

Besides the assessment of resection thickness of femur and tibia using PSI system, there have been several studies comparing CT- and MRI-based PSI system in TKA [17-19]. Two studies [17, 19] compared the accuracy of MRI- and CT-based PSI but they used PSIs from two different manufacturers and different types of the guide. One randomized clinical trial by Pfitzner et al. [17] compared the accuracy of MRI- and CT-based PSI with conventional instrumentation and with each other in TKAs. They found the PSI system increased accuracy compared with conventional instrumentation and that MRI-based PSI was more accurate compared with CT-based PSI, regarding coronal mechanical limb axis, but differences were only subtle and of questionable clinical relevance. And Asada et al. [19] reported that both systems would result in the same accuracy on three planes but high inaccuracy on the sagittal plane on radiographs. While, Silva et al. [18] evaluated the same PSI systems used in our study and reported improved tibial component rotation with MRI-based PSI as compared to CT-based PSI. However, they only assessed postoperative rotation of femur and tibial components. Furthermore, Frye et al. [27] reported better coronal alignment with MRI-based PSI than CT-based PSI. In the current study, the mean coronal and sagittal alignments of the femoral and tibial components were not significantly different between two groups. The rotational alignment of the femoral component has been investigated in several studies. Heyse and Tibesku [28] reported that the incidence of outliers in rotational alignment was remarkably low (2.2\%) in a postoperative MRI analysis of 46 TKAs using an MRI-based PSI, whereas Noble et al. [12] observed outliers in $23.2 \%$ of the 60 knees after TKA using an MRI-based PSI. However, it should be noted that these studies were comparison between the PSI system and the conventional equipment. In the current study, no significant difference was found in the rotational alignment and the incidence of outliers between the MRI and CT groups.

MRI and CT may have their own strengths and limitations when used for creation of PSI system, and a 
discrepancy appears to unavoidably develop between the bone model and the actual shape and size of the patient's femur and tibia due to various stages of measurement and manufacturing processes $[8,9]$. In an initial animal study on PSI system, bone models generated from CT scans have been shown to be more accurate and their external surface boundaries smoother and freer from distorting artifacts than MRI-based models in 10 ovine knees [8]. In contrast, MRI scans enabled more accurate component alignment in knees in which the articular cartilage was worn away than CT scans [9]. And, based on the cartilage surface estimated by the surgeon with CT-based PSI, deviation from the real joint surface is possible, which can result in a poorer fit. However, there was no difference on the three planes between the MRI and CT groups in our study. It is likely that the fitting of the CT-based PSI guide on the articular surface would be sufficient, despite the loss of the contract area.

This study also had several limitations. First, the costeffectiveness of PSI was not assessed. However, the current study mainly focused on the comparison of accuracy between the preoperatively planned cutting thickness and the intraoperative actual cutting thickness in both CT- and MRI-based PSI systems rather than the effectiveness of the system itself. Moreover, as individual countries have different policies concerning the price of using it and different degrees of convenience in terms of using MRI/CT, comparison in such manner would apparently be considered subjective. Second, because the CT-based and MRI-based PSI were the products of the same manufacturer; therefore, it might be difficult to generalize our results to other PSI systems. Third, the number of subjects was relatively small. This study was planned prospectively but, the sample size was not researched to calculated sample size. And finally, we did not evaluate the PSI against conventional instrument in TKA.

Despite the advantage of full visualization of the cartilage layer, the MRI-based PSI did not show better accuracy than the CT-based PSI in terms of planned resection thickness in TKA. Therefore, we recommend that a preoperative decision on whether MRI or CT will be used for creation of PSI should be based on the availability of an imaging modality in the hospital/country system, the risk of radiation exposure and preference of a surgeon or a patient. In addition, we suggest that, in the planning of TKA by using a CT-based PSI system in a varus knee, adjustment should be made in preoperative planning to add approximately $2 \mathrm{~mm}$ of invisible cartilage thickness in determining the extent of resection of the lateral tibial condyle where the cartilage will be relatively well maintained.

\section{Conclusion}

Although MRI provides visualization of cartilage, MRIbased PSI system did not show better accuracy in predicting the thickness of bone resection than CT-based
PSI. Moreover, there was no difference in radiographic findings and clinical outcomes between the two groups. Future studies should compare the two modalities in terms of costs and burden of resources.

\section{Abbreviations}

3D: Three-dimensional; CT: Computed tomography; ICC: Intraclass correlation coefficient; MA: Mechanical axis; MRI: Magnetic resonance imaging; PSI: Patient-specific instrumentation; TKA: Total knee arthroplasty

\section{Acknowledgements}

Not applicable.

\section{Authors' contributions}

All the authors contributed substantially to planning of the study and drafting of the paper. KIK and DGK wrote the paper and conducted the study design. JKB: conducted the data analyses and interpreted the results. All authors read and approved the final manuscript.

\section{Funding}

There was no external source of funding for this study.

\section{Availability of data and materials}

The datasets used and/or analysed during the current study are available from the corresponding author on reasonable request.

Ethics approval and consent to participate

Approval for this study was granted by the institutional review board of Kyung Hee University Hospital at Gangdong (KHNMC 2011-058).

\section{Consent for publication}

Written informed consent was obtained from all patients before surgery.

\section{Competing interests}

The authors declare that they have no competing interests.

Received: 7 August 2019 Accepted: 12 December 2019

Published online: 14 January 2020

\section{References}

1. Papaioannou G, Nianios G, Mitrogiannis C, Fyhrie D, Tashman S, Yang KH. Patient-specific knee joint finite element model validation with highaccuracy kinematics from biplane dynamic roentgen stereogrammetric analysis. J Biomech. 2008;41(12):2633-8.

2. Nunley RM, Ellison BS, Zhu J, Ruh EL, Howell SM, Barrack RL. Do patientspecific guides improve coronal alignment in total knee arthroplasty? Clin Orthop Relat Res. 2012;470(3):895-902.

3. Nunley RM, Ellison BS, Ruh EL, Williams BM, Foreman K, Ford AD, et al. Are patient-specific cutting blocks cost-effective for total knee arthroplasty? Clin Orthop Relat Res. 2012;470(3):889-94.

4. Lombardi AV Jr, Berend KR, Adams JB. Patient-specific approach in total knee arthroplasty. Orthop. 2008;31(9):927-30.

5. Klatt BA, Goyal N, Austin MS, Hozack WJ. Custom-fit total knee arthroplasty (Otisknee) results in malalignment. J Arthroplast. 2008;23(1):26-9.

6. Ng VY, DeClaire JH, Berend KR, Gulick BC, Lombardi AV Jr. Improved accuracy of alignment with patient-specific positioning guides compared with manual instrumentation in TKA. Clin Orthop Relat Res. 2012;470(1):99-107.

7. Spencer BA, Mont MA, McGrath MS, Boyd B, Mitrick MF. Initial experience with custom-fit total knee replacement: intra-operative events and long-leg coronal alignment. Int Orthop. 2009;33(6):1571-5.

8. White D, Chelule KL, Seedhom BB. Accuracy of MRI vs CT imaging with particular reference to patient specific templates for total knee replacement surgery. Int J Med Robot. 2008:4(3):224-31.

9. Tibesku CO, Innocenti B, Wong P, Salehi A, Labey L. Can CT-based patientmatched instrumentation achieve consistent rotational alignment in knee arthroplasty? Arch Orthop Trauma Surg. 2012;132(2):171-7.

10. Conteduca F, lorio R, Mazza D, Ferretti A. Patient-specific instruments in total knee arthroplasty. Int Orthop. 2014;38(2):259-65. 
11. Nam D, Maher PA, Rebolledo BJ, Nawabi DH, McLawhorn AS, Pearle AD Patient specific cutting guides versus an imageless, computer-assisted surgery system in total knee arthroplasty. Knee. 2013;20(4):263-7.

12. Noble JW Jr, Moore CA, Liu N. The value of patient-matched instrumentation in total knee arthroplasty. J Arthroplast. 2012;27(1):153-5.

13. Chareancholvanich K, Narkbunnam R, Pornrattanamaneewong C. A prospective randomised controlled study of patient-specific cutting guides compared with conventional instrumentation in total knee replacement. Bone Joint J. 2013;95-B(3):354-9.

14. Lustig S, Scholes CJ, Oussedik SI, Kinzel V, Coolican MR, Parker DA Unsatisfactory accuracy as determined by computer navigation of VISIONAIRE patient-specific instrumentation for total knee arthroplasty. J Arthroplast. 2013;28(3):469-73.

15. Victor J, Dujardin J, Vandenneucker H, Arnout N, Bellemans J. Patient-specific guides do not improve accuracy in total knee arthroplasty: a prospective randomized controlled trial. Clin Orthop Relat Res. 2014;472(1):263-71.

16. Vundelinckx BJ, Bruckers L, De Mulder K, De Schepper J, Van Esbroeck G. Functional and radiographic short-term outcome evaluation of the Visionaire system, a patient-matched instrumentation system for total knee arthroplasty. J Arthroplast. 2013;28(6):964-70.

17. Pfitzner T, Abdel MP, von Roth P, Perka C, Hommel H. Small improvements in mechanical axis alignment achieved with MRI versus CT-based patientspecific instruments in TKA: a randomized clinical trial. Clin Orthop Relat Res. 2014;472(10):2913-22.

18. Silva A, Pinto E, Sampaio R. Rotational alignment in patient-specific instrumentation in TKA: MRI or CT? Knee Surg, Sports Traumatol, ArthrosC: Official J ESSKA. 2016;24(11):3648-52.

19. Asada S, Mori S, Matsushita T, Nakagawa K, Tsukamoto I, Akagi M. Comparison of MRI- and CT-based patient-specific guides for total knee arthroplasty. Knee. 2014;21(6):1238-43.

20. Kladny B, Bail H, Swoboda B, Schiwy-Bochat H, Beyer WF, Weseloh G. Cartilage thickness measurement in magnetic resonance imaging. Osteoarthr Cartil. 1996;4(3):181-6.

21. Shepherd DE, Seedhom BB. Thickness of human articular cartilage in joints of the lower limb. Ann Rheum Dis. 1999;58(1):27-34.

22. Hoffart HE, Langenstein E, Vasak N. A prospective study comparing the functional outcome of computer-assisted and conventional total knee replacement. J Bone Joint Surg Br. 2012;94(2):194-9.

23. Miller MC, Berger RA, Petrella A, Karmas A, Rubash HE. Optimizing femoral component rotation in total knee arthroplasty. Clin Orthop Relat Res. 2001;392:38-45.

24. Insall JN, Dorr LD, Scott RD, Scott WN. Rationale of the knee society clinica rating system. Clin Orthop Relat Res. 1989;248:13-4.

25. Ware JE, Jr., Sherbourne CD. The MOS 36-item short-form health survey (SF-36). I. Conceptual framework and item selection. Med Care 1992;30(6):473-483.

26. Hafez MA, Chelule KL, Seedhom BB, Sherman KP. Computer-assisted total knee arthroplasty using patient-specific templating. Clin Orthop Relat Res. 2006;444:184-92

27. Frye $B M$, Najim AA, Adams JB, Berend KR, Lombardi AV Jr. MRI is more accurate than CT for patient-specific total knee arthroplasty. Knee. 2015; 22(6):609-12.

28. Heyse TJ, Tibesku CO. Improved femoral component rotation in TKA using patient-specific instrumentation. Knee. 2014;21(1):268-71.

\section{Publisher's Note}

Springer Nature remains neutral with regard to jurisdictional claims in published maps and institutional affiliations.

Ready to submit your research? Choose BMC and benefit from:

- fast, convenient online submission

- thorough peer review by experienced researchers in your field

- rapid publication on acceptance

- support for research data, including large and complex data types

- gold Open Access which fosters wider collaboration and increased citations

- maximum visibility for your research: over $100 \mathrm{M}$ website views per year

At $\mathrm{BMC}$, research is always in progress.

Learn more biomedcentral.com/submissions 